\title{
Generation of Radially Polarized Beam for Laser Micromachining
}

\author{
Krishnan Venkatakrishnan ${ }^{1}$ and Bo $\operatorname{Tan}^{2}$ \\ 1:Department of Mechanical and Industrial Engineering 2 Department of Aerospace Engineering \\ Ryerson University, 350 Victoria Street, Toronto, Canada M5B $2 K 3$ \\ venkat@ryerson.ca;tanbo@ryerson.ca
}

\begin{abstract}
A novel means of generating radially polarized light is reported in this paper. A birefringent plate was placed in a divergent or divergent portion of the laser beam for polarisation conversation. The influence of birefringent plate thickness on the purity and efficiency of radial beam conversion is studied qualitatively. This technique is robust, simple and cost effective for radial polarisation conversion, and involvs only minimal optical components compared to alternative methods. Comparative analysis between linearly and radially polarized beams at different experimental condition is reported. Radially polarized beam increases the machining efficiency by $40-100 \%$ and reduces the focused spot size by more than $30 \%$. Radially polarized beam finds wide application in semiconductor manufacturing. Application of radially polarized light for interconnect via drilling is demonstrated. DOI:10.2961/jlmn.2012.03.0008
\end{abstract}

Keywords: radial polarization, micromachining

\section{Introduction}

Polarization state of the laser beam is one of the important parameters of laser radiation for micromachining. Conventionally, linear and circularly polarisation state is used for all semiconductor laser processing applications, including interconnect via drilling. Radial polarisation is found to generate small focused spot size and increased absorption rate and hence high efficiency in ablation of semiconductor materials. The only few published research work that investigated the effect of radial polarization on laser machining[1-2] have proven that radial polarization significantly reduces the feature size and drastically improves machining quality and efficiency. Although both experimental and theoretical analysis shows that radially polarized beam has significant advantages over conventional linearly polarized laser beam, the generation of radial polarization is problematic, which prevents the employment of radially polarized laser beam from real world micromachining applications.

Radially polarized light beam is, in general, generated by two means, namely active conversion and passive conversion. In the former method, the polarization is rotated into radial state inside a laser cavity by a discontinuous phase element[3-10], a specially designed optical element. Active conversion has the advantages of better polarization purity. However, these lasers have to operate near instability region, limiting light throughput. Also, these optical elements are usually cumbersome operate and difficult to implement. The passive conversion is to convert emitting linearly polarized laser beam into radially polarized beam using either unconventional optics, such as specially designed polarization converter and axicon lenses [11-14], or complex optic configurations[15]. This method requires rigorous alignment or special fabrication techniques, which makes it impractical and difficult to materialize in reality.

In this paper, we report a practical means of generating radially polarized beam by passive conversion using birefringent plate. The advantages of micromachining with radially polarized laser beam were demonstrated through interconnect vias drilling.

\section{Generation of radial Polarization}

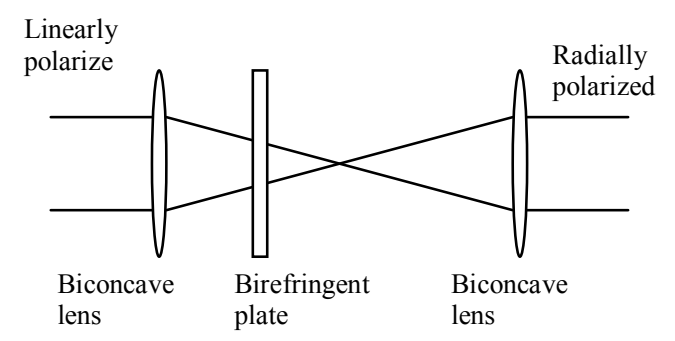

Fig.1. Polarization converter with birefringent plate in between a Keplerian telescope.

Figure 1 shows a novel yet simple technique for generating radial polarization light from a linearly or circularly polarized laser light. A birefringent plate of predetermined thickness is placed in between a telescopic module, either Keplerian type or Galilean type. Light rays of a convergent beam or divergent beam travel different optical path lengths when they transmit a birefringent plate. The light rays at the central part of the beam travel shorter distance than those at the edge through a birefringent material. This result in shift in the electric vector in the plane of the beam cross-section is parallel to the radial direction.

Consequently, the polarization state is partially modulated into radial polarisation state depending on the convergence or divergence angle of the laser beam and the thickness/properties of the birefringent plate. This shift in polarisation state can be explained by Ambrus KohaziKis's [14] study on the reflection or refraction of laser light on interface of isotropic interference of focused light beam. 


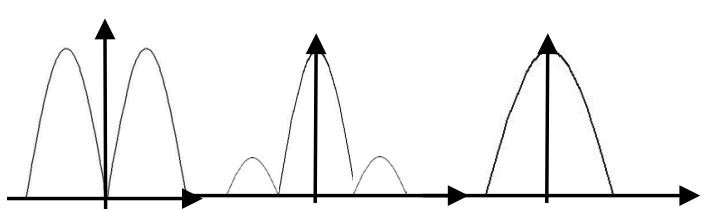

(a)

(b)

(c)

Fig.2. Intensity profile of radially polarized laser beam (a) near field (b) far field $\mathrm{X}$ axis (c) far field $\mathrm{Y}$ axis.

According to prior research, radially polarized beam consists of two orthogonally polarized TEM01 modes. The resultant near field beam is hollow: the intensity distribution is axially symmetric and having a doughnut mode. Since the two orthogonally polarized TEM01 beams are in phase, a high peak should present at focus. The intensity pattern in near field and far field is given in Figure 2. An image of the laser beam after passing the optical set shown in Figure 1 and before it is focused onto the target surface. The waveplate used for this study is a standard crystal quartz waveplate supplied by CVI Melles Griot. The theory that a birefringent plate on a convergent beam converts the polarization state will depend on numerical analysis and simulation. We will carried out this part of work in future and report it separately.

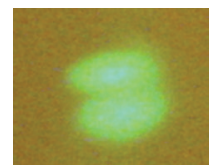

Figure 3 Image of beam profile
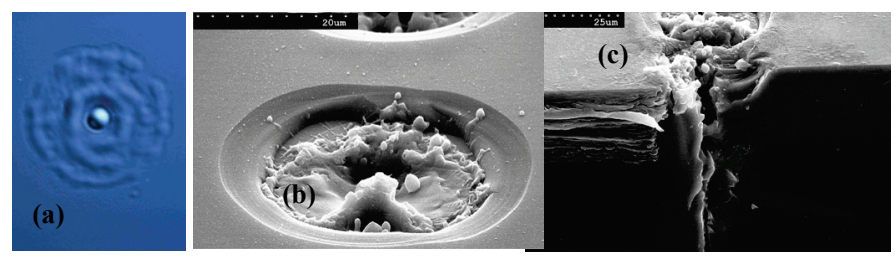

Figure 4 (a) ablated crater at $1 \mathrm{~mm}$ above focal point. (b)SEM image of the machined spot (c) SEM image of the profile of the machined spot reveals high intensity concentration at the center of the laser spot. (All images at $\times 3000$ magnification)

For a conventional linearly polarized beam, the electric field is transverse at the focus. whereas, the electric field of an axicon laser beam with radial polarization leads to a longitudinal electric field (parallel to the direction of propagation) at the focus. The spot size for this radially polarized field is smaller than for linear polarization. Theoretical limit of radially polarized field is calculated as $0.16 \mu \mathrm{m}$ well below $0.26 \mu \mathrm{m}$ for linear polarized light and $0.22 \mu \mathrm{m}$ for circularly polarized light under the same experimental condition [16]

The intensity profile of the convergent or divergent beam that transmits the birefriengent plate was verified by observation of features ablated with the beam. A diode- pumped solid state Nd-YAG laser at $355 \mathrm{~nm}$ wavelength of $11 \mathrm{~W}$ average output power was used to provide the linearly polarized incident beam. The test samples were polished silicon substrates with crystal orientation of (111). A singlet lens of $100 \mathrm{~mm}$ focal length focuses the modulated beam into a small focused laser spot. In order to study the intensity distribution at near filed, silicon substrate was positioned at $1 \mathrm{~mm}$ away from focal point The laser pulse energy and the out of focal distance were chosen such that there is surface modification rather than ablation. Optical image of the modified surface is given in Figure 4(a). The high peak at the center of ablated crater suggests that the laser spot has ring intensity distribution pattern. The silicon substrate was then moved to the focal point. Laser pulses were fired at pulse energy of $300 \mu \mathrm{J}$ at $4 \mathrm{kHz}$ repetition rate for 2 pulses so that microholes could be ablated into the material. The substrate was then mechanically cleaved to reveal the profile of the microhole. The sample was observed with Scanning Electronic Microscopy (SEM). The resultant image, given in Figure 4(b) and (c), shows a stepped hole, with a deep sharp hole in the center surrounded by sallow annular. The morphology of the ablated features indirectly proved that the intensity pattern of the output beam consists to intensity profile of radially polarized beam obtained by other researchers $[17,18]$.

In order to further validate the polarization state after transmitting divergent beam through birefringent plate, comparison experiments were carried out with birefringent plate placed in the path of converging/diverging beam and along a collimated beam at the same experimental conditions. Microvias were punched at different pulse energy by varying the repetition rate of the laser beam from $80 \mathrm{kHz}$ to $120 \mathrm{kHz}$, and the result at $120 \mathrm{kHz}$ for one pulse ablation at focal point is shown in Figure 5. When the birefringent plate is placed along the convergent or divergent path of the laser beam, it resulted in smaller ablated spot size and increased ablation depth due to shift in the laser polarization state to radial. Presence of birefringent plate along the collimated beam made no noticeable difference in the ablated feature size or depth compared to ablation using linearly/circularly polarized laser beam. This further proves the phenomenon for conversation of polarization state by a simple and practical method using birefringent plate.

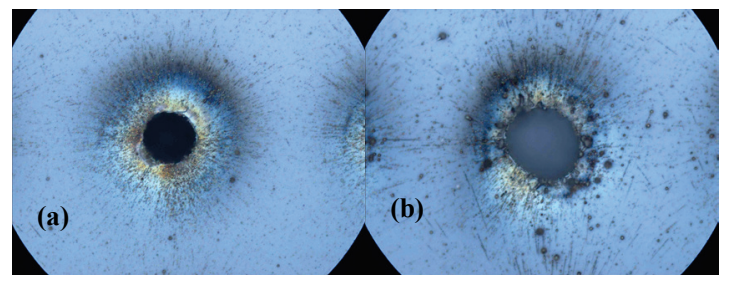

Figure 5 Microhole ablated with birefrengent plate placed at (a) Convergent or divergent beam path and (b) collimated beam path. $(\times 3000$ magnification $)$ 


\section{Effect of incident polarization}

To study the effect of incident polarization state of the laser on the polarization conversion (To Radial state), experiment was conducted at $80 \mathrm{kHz}$ repetition rate at a pulse energy of $245 \mu \mathrm{J}$. Silicon substrate was ablated with single laser pulse. The Input laser beam polarization to the birefringent crystal was varied from linear ( $S$ and $P$ polarization) and circular polarization. The experimental results revealed that incident polarization state had no effect on the ablated micro via. The diameter and depth of the micro via and the quality of the micro via remains consistent regardless of the input polarization state. This may suggest that the purity or degree of radial polarization remains the same for liner and circular state of incident laser beam.

\section{Effect of plate thickness}

To study the effect of bifriengent plate thickness on the conversion efficiency, plates of thickness varied from 0.5 $\mathrm{mm}$ to $2.5 \mathrm{~mm}$ were place along a divergent beam path generated by a plano-concave lens of $100 \mathrm{~mm}$ focal length. The output beam was was focused using a telecentric lens of $100 \mathrm{~mm}$ focal length to a spot size of $15 \mu \mathrm{m}$. Microholes were ablated with pulse energy of $280 \mu \mathrm{J}$ at $40 \mathrm{kHz}$ pulse repetition rate and a dwell time of $200 \mu$ s (corresponding to 8 laser pulses). The depth and diameter of the produced vias were measured and is plotted in Figure 6 and Figure 7, respectively. The via diameter decreased from $40 \mu \mathrm{m}$ to 30 $\mu \mathrm{m}$ with the increase in the birefringent plate thickness and remains constant above $2 \mathrm{~mm}$ birefringent plate thickness. Similarly, the ablated via depth increased from $40 \mu \mathrm{m}$ to 58 $\mu \mathrm{m}$ with the increase in the birefringent plate thickness and remains constant above $1.5 \mathrm{~mm}$ plate thickness. It is reasonable to assume that with the increase in birefringent plate thickness, the degree of radial polarization increases since the laser beam transmits longer distance within the birefringent plate, consequently, more efficient conversion. It is suspected that the thickness of the birefringent plate for optimal conversion depends on the convergence or divergence angle of the laser beam and the collimated beam diameter. Research work is currently carried out to confirm such assumption and the results will be reported shortly. For all our further experiments, we used $2.5 \mathrm{~mm}$ birefringent plate thickness to generate beam with higher degree of radial polarization.

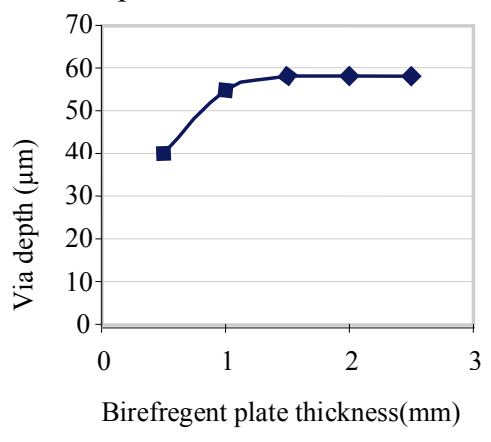

Fig. 6. Effect of plate thickness on via depth

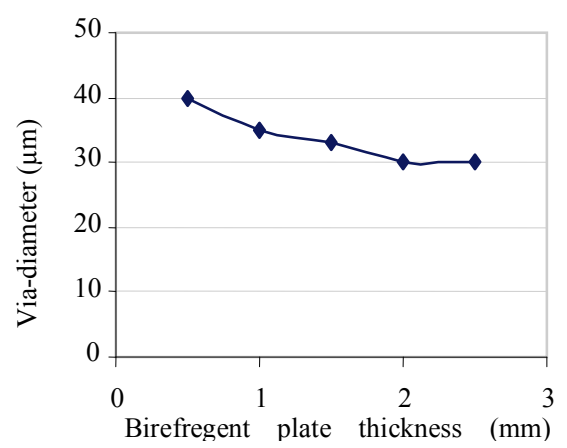

Fig. 7. Effect of plate thickness on via diameter

\section{Comparison of via drilling with linearly and radially polarized beam}

Experiment was carried out at linear and radial polarisation state for pulse energy of $50 \mu \mathrm{J}$ at $50 \mathrm{kHz}$, $80 \mathrm{kHz}$ and $100 \mathrm{kHz}$ pulse repetition rate. The resultant vias are compared side by side in Figure 8 . For all three sets of machining parameters, vias ablated by radially polarized beam are superior to those ablated by linearly polarized beam. Not only do they have reduced diameter and extended depth, but also they present much cleaner finish and less deformation in shape.

The advantage of radial polarization for material breakdown comes in two fold. First for all, the presence of the longitudinal electric field at the focus makes the radially polarized beam has maximum resonance absorption[17, 18]. The effective absorption power of radially polarized beam is nearly twice that of linearly or circularly polarized beam and hence we notice a $30-100 \%$ increase in via depth at radial polarization state compared to linearly polarization state at same experimental conditions. As photonic energy is more efficiently absorbed by material, damages that usually associated with laser ablation due to thermal heating will subside. Therefore, better finishing quality could be expected with radially polarized beam. This explains the cleaner finish on the top surface (less debris deposition). Secondly, the fluency of the laser spot focused from radialy polarized beam is higher than that from linearly polarized beam since radially polarized beam has a reduced spot size, as discussed in prior section. Therefore, higher machining efficiency could be expected with the same laser power. 


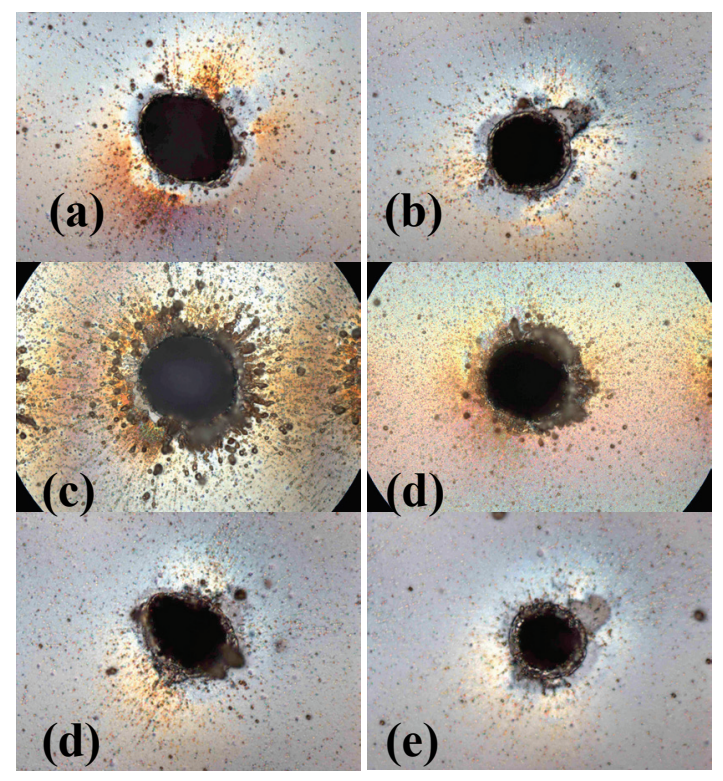

Figure 8(a) (c) (d) Microvias drilled with linearly polarized beam at $50 \mathrm{kHz}, 80 \mathrm{kHz}$, and $100 \mathrm{kHz}$ repetition rate, respectively. (b) (d) (e) Microvias drilled with radially polarized beam at $50 \mathrm{kHz}, 80 \mathrm{kHz}$, and $100 \mathrm{kHz}$ repetition rate, respectively. (All images at $\times 3000$ magnification)

As for via drilling an additional advantage of radially polarized beam is that the electric vector at focus is axially symmetric. Thus, photonic energy deposition on the material surface and light reflection inside the via channel are equal at all direction, which helps maintain the roundness of microvia. On the contrary, the absorption and the reflection of $\mathrm{p}$ - and s-polarized light are unequal, imposing challenges on produce well-shaped vias. The experiemental results presented in Figure 8 shows that those vias produced by linearly polarized beam are more or less out of shape, whereas, those produce by radially polarized beam are of better roundness.

\section{Application of radially polarized beam for microvia drilling}

Interconnect via drilling using radial polarized beam can shrink via diameter by $30-40 \%$ compared to liner polarized beam and hence can increase interconnect via density considerably. The reduced via diameter without modifying the optical parameter increases the scan field and position accuracy at radially polarized light compared to linear polarization state for a given focus length. Also, interconnect via drilling efficiency can be increased by 30 $100 \%$ at radial polarization state and hence bring the cost of manufacturing considerably. Profile of a via drilling with radially polarized beam at $80 \mathrm{kHz}$ for $50 \mu \mathrm{m}$ is presented in Figure 9. The top surface and side wall are of good quality (after pressurised water wash).

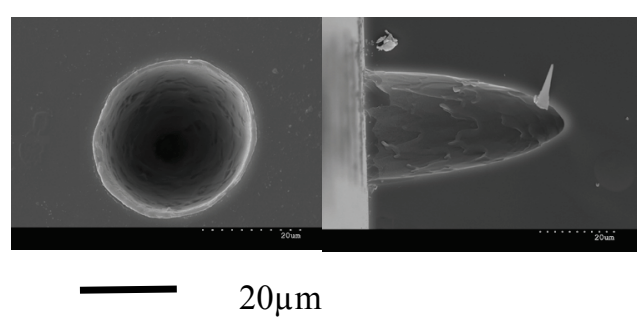

Figure 9 Top surface and sidewall of microvia ablated with radially polarized laser beam

\section{Conclusion}

Radial polarized beam was generated from a linear or circular polarized beam by using a birefringent plate of required thickness in a convergent or divergent path of a laser beam. Since the technique involves only one optical component, the process is reliable and the generated radially polarized beam is stable in polarization state and power. It can be easily implemented with commercially available berifringent plate at minimal cost. The novel radial polarisation conversion technique will find wide application in measurement and machining application, where there is a need to focus the laser beam tighter and to increase the machining throughput and improve ablation quality. For instance, it could be applied for semiconductor manufacturing process, such as thin wafer dicing, interconnection drilling, modification of integrated circuit, and resister trimming, just to name a few. We had demonstrated that if used for micro via drilling it will significantly increase the interconnection density and improve the machining throughput with better machining quality.

\section{Acknowledgement}

This research work was supported by Ryerson University and Natural Science and Engineering Research Council of Canada.

\section{References}

1. V. G. Niziev and A. V. Nesterov, Influence of beam polarization on laser cutting efficiency, J. Phys. D: Appl. Phys. 32 (1999), 1455-61.

2. M. Meier, V. Romano, and T. Feurer, Material processing with pulsed radially and azimuthally polarized laser radiation, Appl. Phys. A. 86, 329-334 (2007).

3. T. Moser, H. Glur, V. Romano, F. Pigeon, O. Parriaux, M. A. Ahmed and T. Graf, Polarization-selective grating mirrors used in the generation of radial polarization, Appl. Phys. B 00, 1-7 (2005).

4. G. Volpe and D. Petrov, Generation of cylindrical vector beams with few-mode fibres excited by LaguerreGaussian beams, Optics Commmunications, 237 (2004), 89-95.

5. R. Oron, L, Shimshi, S.Blit, N. Davidson, A. Friesem, and E. Hasman, Laser operation with two orthogonally polarized transverse modes, Applied Optics, Vol. 41, No. 18, 2002, 3634-3637.

6. Goncharshii, A. V. Nesterov, V. V. Nizev, L. V. Novikova, and V. P. Yakunin, Optical elements of a laser 
cavity for the production of a beam with axially symmetric polarization, Optics and Spectroscopy, Vol. 89, No. 1. (2000), 146-149.

7. Tovar, Production and propagation of cylindrically polarized Laguerre-Guassian laser beams, J. Opt, Soc. Am. A, 15 (1998), 2705-11.

8. Olson, P. L. Greene, G. W. Wicks, D. G. Hall and S. Rishton, High-order azimuthal spatial modes of concentric-circle-grating surface-emitting semiconductor lasers, Appl. Phys. Lett., 72(11), 1998, 1284-1286.

9. Y. Kozawa and S. Sato, Generation of a radially polarized laser beam by use of a conical Brewster prism, Optics Letters, Vol. 30, No. 22, (2005), 3063-3065.

10. Goncharskii, A. V. Nesterov, V. G. Nizev, L. V. Novikova, and V. P. Yakunin, Optical elements of a laser cavity for the production of a beam with axially symmetric polarization, Optics and Spectroscopy, 89(1), 2000, 146-9.

11. R. Oron, S. Blit, N. Davidson and A. Friesem, The formation of laser beams with pure azimuthal or radial polarization, Applied Physics Letters, Vol. 77, No. 22, (2000), 3322-3324

12. M. Stalder and M. Schadt, Linearly polarized light with axial symmetry generated by liquid-crystal polarization converters, Optics Letters, 21(3A), 1996, 1948-1950.

13. J. Armstrong, M. C. Phillips, and A. V. Smith, Generation of radially polarized beams with an imagerotating resonator, Applied Optics, 42(18), 2003, 35503554.

14. K. Hazi-Kis, Cross polarization effects of light beams at interfaces of isotropic media, A GAMF kozlemenyei, Kecskemet, X X, Evfolam (2004).

15. S. C. Tidwell, G. H. Kim and W. D. Kimura, Efficient radially polarized laser beam generation with a double interferometer, Applied Optics, Vol. 32, No. 27,

(1993), 5222-5228.

16. S. Quabis, R. Dorn, M. Eberler, O. Glockl, and G. Leuchs, Focusing light to a tighter spot, Optics Communications, 179 (2000), 1-7.

17. A.V.Nesterrov and V.G Niziev, Laser beam with axially symmetric polarisatoin, J. Phys. D, Appl. Phys. 33 (2000), 1817-1822.

18. R. Dorn, S. Quabis and G. Leuchs "Sharp focus for radially polarized light beam" Phys. Rev. Lett., Vol 91, No. 23, 2003.

(Received: May 01, 2012, Accepted: August 30, 2012) 